\title{
A GLOBAL THEORY OF STEADY VORTEX RINGS IN AN IDEAL FLUID
}

\author{
BY L. E. FRAENKEL AND M. S. BERGER ${ }^{1}$
}

Communicated by Hans Weinberger, December 18, 1972

The question of whether the equations governing the motion of an inviscid, incompressible fluid admit solutions representing steady vortex rings has not been studied widely, despite the central place of such rings in the theory of vortex motion initiated by Helmholtz [1] in 1858. Hill [2] discovered in 1894 an explicit particular solution for which the 'ring' is actually a ball in $\boldsymbol{R}^{3}$. More recently, there have appeared local existence proofs for (a) steady rings of small cross-section [3], [4], [5] and (b) steady rings close to Hill's vortex, but homeomorphic to a solid torus [6]; these two cases represent opposite extremes. The present note outlines what we believe to be the first global existence theory for steady vortex rings.

By a steady vortex ring we mean a figure of revolution $\mathscr{A}$ that is expected to be homeomorphic to a solid torus in most cases, and is associated with a continuous, axi-symmetric, solenoidal vector field $q$ (the fluid velocity) defined in a cylinder $V$ or in the whole of $\boldsymbol{R}^{3}$, and having the following properties when we take axes fixed in the ring $\mathscr{A}$. (a) Both $\mathscr{A}$ and $\boldsymbol{q}$ do not vary with time; (b) the vorticity $\boldsymbol{\omega} \equiv \operatorname{curl} \boldsymbol{q}$ has positive magnitude in $\mathscr{A}$, vanishes in $V-\mathscr{A}$ or $\boldsymbol{R}^{3}-\mathscr{A}$, and satisfies a nonlinear equation of motion which, among other things, determines the boundary of $\mathscr{A}$; (c) $\boldsymbol{q}$ has a prescribed normal component on $\partial V$, or tends to a constant value at infinity in $\boldsymbol{R}^{3}$. For the case of a cylinder $V$ these requirements lead to equations (1) and (2) below, and we solve the resulting problem by means of the calculus of variations in the large [7], [8]. We also use Steiner symmetrization [9], the generalized maximum principle $[10]$ and certain a priori estimates to describe the solution and to deal with two limiting cases: (i) that when the nonlinear term in the differential equation ( $2 \mathrm{a}$ ) is discontinuous, and (ii) that when the domain of $\boldsymbol{q}$ is the whole of $\boldsymbol{R}^{3}$ (so that the usual compactness theorems, needed for the solution of variational problems, do not hold).

1. Preliminaries. Let $X=\left[X_{1}, X_{2}, X_{3}\right]=[r \cos \theta, r \sin \theta, z]$ be a point of $\boldsymbol{R}^{3}$, so that $(r, \theta, z)$ are cylindrical coordinates. Consider the axi-symmetric flow of an inviscid fluid, of uniform density $\rho$, in a cylinder $V$ that is here represented in a meridional plane $(\theta=$ const $)$ by the domain $D$;

AMS (MOS) subject classifications (1970). Primary 35J60, 76C05.

${ }^{1}$ Research partly sponsored by AFOSR 73-2437, and an NSF grant. 


$$
V=\left\{X\left|X_{1}^{2}+X_{2}^{2}<a^{2},\right| X_{3} \mid<b\right\} \text { and } D=\{(r, z)|0<r<a,| z \mid<b\} .
$$

We seek a stream function

$$
\Psi(r, z)=\psi(r, z)-\frac{1}{2} W r^{2}-k \quad(W=\text { const }>0, k=\text { const } \geqq 0),
$$

such that the velocity vector field $q$ has components $\left(-\Psi_{z} / r, 0, \Psi_{r} / r\right)$ in the directions $(r, \theta, z)$ increasing; $\psi$ is to be the stream function of the velocity field induced by a steady vortex ring; $-\frac{1}{2} W r^{2}$ represents a uniform stream; and $k$ is a flux constant which is zero for Hill's vortex and very large for rings of small cross-section.

The problem of finding a steady vortex ring in $V$ may be formulated as follows. Given $W, k$, a vorticity function $f$ as in (3) below, and the kinetic energy $\pi \rho \eta$ of the desired vortex motion, we seek a vortex stream function $\psi$ satisfying

$$
r\left(\psi_{r} / r\right)_{r}+\psi_{z z}=-\lambda r^{2} f(\Psi) \quad \text { in } D,\left.\quad \psi\right|_{\partial D}=0,
$$

where $\Psi$ and $\psi$ are related by (1), and the vortex-strength parameter $\lambda$ is to be such that

$$
\iint_{D} \frac{1}{r^{2}}\left(\psi_{r}^{2}+\psi_{z}^{2}\right) r d r d z=\eta>0 .
$$

The function $f: \boldsymbol{R} \rightarrow[0, \infty)$ is assumed to be nondecreasing and (in the first instance) locally Hölder continuous; more precisely,

$$
f(t)=0 \quad \text { for } t \leqq 0, \quad f(t)>0 \text { for } t>0,
$$

and

(3c) $0 \leqq f(t)-f(s) \leqq \frac{1}{2} M^{\mu}\left\{1+(M t)^{m-1}\right\}(t-s)^{\mu} \quad$ for $t \geqq s \geqq 0$,

where $M>0, m \geqq 1$ and $\mu \in(0,1)$ are given constants.

One can avoid the singularity of the differential equation (2a) at $r=0$ by considering in place of $\psi$ the vector potential $\alpha(X)=[-\psi \sin \theta / r$, $\psi \cos \theta / r, 0]=\left[\alpha_{1}, \alpha_{2}, 0\right]$, where square brackets denote vector components in the directions $X_{j}$ increasing. Then $\psi=X_{1} \alpha_{2}-X_{2} \alpha_{1}$, and in place of (2a) one has the system

(4) $\Delta\left[\alpha_{1}, \alpha_{2}, 0\right]=-\lambda\left[-X_{2}, X_{1}, 0\right] f(\Psi)$ in $V \quad\left(\Delta=\sum_{j=1}^{3} \frac{\partial^{2}}{\partial X_{j}^{2}}\right)$.

Let $H(D)$ denote the Hilbert space that results from completion of the set $C_{0}^{\infty}(D)$ in the norm implied by the inner product

$$
\langle u, v\rangle=\iint_{D} \frac{1}{r^{2}}\left(u_{r} v_{r}+u_{z} v_{z}\right)
$$


the element $d \tau=r d r d z$ of measure being implied wherever no other element is written. The space $H(D)$ is a slight variant of the Sobolev space $W_{1,2}(D)$, and is a natural setting for the problem (2); it is embedded compactly in the space $L_{p}(D, \tau)$ normed by $\left\{\iint_{D}|u|^{p}\right\}^{1 / p}, p \geqq 1$.

2. The solution for continuous vorticity and a bounded domain. We define: $x=(r, z)$ and $S(\eta)=\left\{u \in H(D) \mid\|u\|^{2}=\eta\right\}$. A generalized solution of problem (2) is an element $\psi \in S(\eta)$ and a number $\lambda$ such that

$$
\langle\varphi, \psi\rangle=\lambda \iint_{D} \varphi f(\Psi) \text { for all } \varphi \in H(D) .
$$

This condition characterizes a critical point $\psi$ of the restriction to the sphere $S(\eta)$ of the functional

$$
J(u)=\iint_{D} F\left(u(x)-\frac{1}{2} W r^{2}-k\right), \quad \text { where } F(t)=\int_{0}^{t} f(s) d s .
$$

Note that the integral over $D$ need be taken only over the set

$$
A_{u}=\left\{x \mid u(x)>\frac{1}{2} W r^{2}+k\right\},
$$

which becomes the cross-section $A_{\psi}$ of the vortex ring when $u=\psi$, and similarly in (6). A variational argument [7], [8] and Steiner symmetrization [9] about the line $z=0$, of any function possibly maximizing $J$ on $S(\eta)$, then lead to

THEOREM 1. The variational problem $\max J(u)$ over $S(\eta)$ has a solution $\psi$ that satisfies (6) for a certain $\lambda \in(c, C)$, where the positive bounds $c$ and $C$ depend only on the data of the problem. Moreover, $\psi \in C^{2+\mu}(\bar{D})$ (where $\mu$ is the Hölder exponent of $f)$, with $\psi=0\left(r^{2}\right)$ and $\psi_{r}=0(r)$ for $r \rightarrow 0$, and $\psi$ satisfies (2) pointwise. Finally, $\psi$ is an even function of $z$, is strictly positive in $D$, and has $\psi_{z}<0$ in $D_{+}=\{x \in D \mid z>0\}$.

Here the strict inequality $\psi_{z}<0$ in $D_{+}$is obtained by proving that $\partial \alpha_{2} / \partial z$ is weakly subharmonic in $V^{\prime}=\left\{X \in V \mid X_{1}>0, X_{3}>0\right\}$ and by appeal to generalized maximum principle [10]. Moreover, if the function $f$ is $C^{\prime}$ and convex, the set $A_{\psi}$ is simply connected.

3. The case of discontinuous vorticity. Let $f(t)$ have a simple discontinuity at $t=0$, corresponding to a jump in vorticity at the boundary $\partial A_{\psi}$ of the cross-section of the vortex ring. The only change in (3) is that we weaken the qualification in (3c) to $t \geqq s>0$; then the limit $f(0+)$ exists, and we suppose that the inequality $f(t) \leqq 1+(M t)^{m}, t>0$, still holds.

THEOREM 2. If $f(t)$ has a simple discontinuity at $t=0$, there still exists 
a pair $(\psi, \lambda)$ as follows. The function $\psi \in C^{1+v}(\bar{D})$ for any $v \in(0,1)$, and maximizes $J$ on $S(\eta)$; the constant $\lambda$ is bounded as in Theorem 1 ; and, apart from failing to satisfy (2a) on the set $\Psi^{-1}(0)$, which has plane measure zero, $\psi$ is a pointwise solution of the problem (2).

One proves this theorem by taking a sequence $\left\{f_{n}\right\}$ of smooth approximations to $f$, such that $f_{n} \rightarrow f$ as $n \rightarrow \infty$. The corresponding solutions $\left\{\left(\psi_{n}, \lambda_{n}\right)\right\}$ form a relatively compact set in $C^{1+v}(\bar{D}) \times \boldsymbol{R}$, and, resorting to a subsequence if necessary, we call the limit point $(\psi, \lambda)$. It is clear that $\Psi_{z}=\psi_{z} \leqq 0$ in $\bar{D}_{+}$, and the generalized maximum principle [10] implies once more that $\Psi_{z}<0$ in $D_{+}$. This is the crucial result, for it shows that the set $\Psi^{-1}(0)$ has measure zero, and allows us to prove that

$$
\psi\left(x_{0}\right)=\lambda \iint_{D} G\left(x_{0}, x\right) f(\Psi(x))
$$

(where $G$ is the Green function of the linear analogue of $(2 a, b)$ ), by passing to the limit in the corresponding expression for $\psi_{n}$.

4. The unbounded flow field. We continue to allow the possibility of a simple discontinuity of $f(t)$ at $t=0$. Let $\Pi$ be the half-plane $\{r>0$, all $z$, and let $H(\Pi)$ and $L_{p}(\Pi, \tau)$ be defined by the statements in $\S 1$, with $\Pi$ replacing $D$. Sets bounded in $H(\Pi)$ need not be bounded, let alone compact, in $L_{p}(\Pi, \tau)$. However, in all cases of our problem the values of $\psi$ on the set $A_{\psi}$ (where $\Psi(x)>0$ ) determine $\psi$ everywhere by means of (8). Therefore it suffices to prove existence of a bounded domain $\Omega$, independent of the lengths $a$ and $b$ defining $D$, such that $A_{\psi}$ remains in $\Omega$ no matter how large $a$ and $b$ become. For then one can consider a sequence $\left\{D_{n}\right\}$ of domains tending to $\Pi$, extract from the corresponding sequence $\left\{\left(\psi_{n}, \lambda_{n}\right)\right\}$ a subsequence convergent in $C^{1+v}(\bar{\Omega}) \times \boldsymbol{R}$, and finally use the analogue of $(8)$ for the half-plane to extend the limit function $\psi$ from $\bar{\Omega}$ to $\bar{\Pi}$. In this way we obtain

TheOREM 3. Replace $D$ by $\Pi$ in (2) and (7), and demand that $\psi \rightarrow 0$ as $r^{2}+z^{2} \rightarrow \infty$. The problem (2), so modified, still has a solution $(\psi, \lambda)$ with the properties established in Theorem 1 if $f$ is continuous, or in Theorem 2 otherwise. The cross-section $A_{\psi}$ of the vortex ring lies in a bounded domain

$$
\Omega=\left\{(r, z)\left|0<r<r_{*},\right| z\left|<z_{*},\right| z \mid<\frac{\eta}{W\left(W r^{2}+4 k\right)}\right\},
$$

where $r_{*}$ and $z_{*}$ can be estimated in terms of $f, W, k$ and $\eta$, and $z_{*}$ is independent of $k$.

\section{BIBLIOGRAPHY}

1. H. von Helmholtz, Uber Integrale der hydrodynamischen Gleichungen, welche den 
Wirbelwegungen entsprechen, Crelle's Journal 55 (1858), 25-55.

2. M. J. M. Hill, On a spherical vortex, Phil. Trans. Roy. Soc. London A185 (1894), 213-245.

3. L. Lichtenstein, Über einige Existenzprobleme der Hydrodynamik, Math. Z. 23 (1925), 89-154.

4. K. Maruhn, Über die Existenz stationärer Bewegungen von Wirbelringen, Proc. Ninth International Congress Appl. Mech., University of Brussels 1 (1957), 173-176.

5. L. E. Fraenkel, On steady vortex rings of small cross-section in an ideal fluid, Proc. Roy. Soc. London A316 (1970), 29-62.

6. J. Norbury, A steady vortex ring close to Hill's spherical vortex, Proc. Cambridge Philos. Soc. 72 (1972), 253-284.

7. M. M. Vainberg, Variational methods for the study of nonlinear operators, Holden-Day, San Francisco, 1964. MR 31 \#638.

8. M. S. Berger, Lectures on nonlinear problems of mathematical analysis (to appear).

9. G. Pólya and G. Szegö, Isoperimetric inequalities in mathematical physics, Annals of Math. Studies, no. 27. Princeton Univ. Press, Princeton, N.J. 1951. MR 13, 270.

10. W. Littman, Generalized subharmonic functions: Monotonic approximations and an improved maximum principle, Ann. Scuola Norm. Sup. Pisa (3) 17 (1963), 207-222. MR 31 \#1450.

Department of Applied Mathematics and Theoretical Physics, University of Cambridge, Cambridge, England

School of Mathematics, Institute for Advanced Study, Princeton, New Jersey 08540

Current address (M. S. Berger): Department of Mathematics, Belfer Graduate School, Yeshiva University, New York, New York 10033 